\title{
Echinodermata das praias de Salvador (Bahia, Brasil)
}

\author{
Orane Falcão de Souza Alves ${ }^{1}$ \\ Walter Ramos Pinto Cerqueira ${ }^{2}$
}

\begin{abstract}
The Echinoderms of Salvador beaches (Bahia, Brazil). This paper presents 28 species of Echinoderms collected on 5 beaches of Salvador (12 $54^{\prime}$ 'to $13^{\circ} 01^{\prime} \mathrm{S}$ and $38^{\circ} 26^{\prime}$ to $38^{\circ} 33^{\prime} \mathrm{W}$ ), Brazil, which are distributed in 19 families. Ophiuroidea represented $53,6 \%$ of the collected species, followed by Echinoidea $(28,6 \%)$, Asteroidea ( $7,1 \%)$, Holothuroidea $(7,1 \%)$, and Crinoidea $(3,6 \%)$. Ophiuroidea and Echinoidea were the most frequent groups, occurring at all the studied beaches while Crinoidea occurred only on $20 \%$ of them. Most of the species are characterized as belonging to the tropical warm waters, some to the shallow coastal areas and some having a broad bathymetric distribution. The richness of species values on beaches ranged from 7 to 24 , at Itapuã Beach, and from 2 to 14 among different kinds of habitats, where protected ones showed higher values.

KEY WORDS. Echinodermata, beaches, Salvador, Brazil
\end{abstract}

Os estudos realizados com a fauna de equinodermes no estado da Bahia abrangeram basicamente as regiões sul e sudeste, onde está localizado o Banco dos Abrolhos (Verrill 1868; TOMMASI \& ARON 1988; AlbuQuerQue \& GUILE 1991; MANSO 1993), permanecendo a fauna do restante do litoral baiano, inclusive de Salvador, pouco conhecida. RATHBUN (1879) cita a ocorrência de equinodermes na Baía de Todos os Santos. Segundo LANA et al. (1996), uma fração significativa da bibliografia mais recente da costa nordeste do Brasil não foi publicada, e o bentos da região entre Salvador e Vitória é o menos conhecido, tanto de ambientes costeiros como de plataforma.

A orla marítima de Salvador constitui-se num ambiente praial de alta energia. Suas praias são constituídas basicamente por rochas profundamente intemperizadas e a granulometria do sedimento corresponde à areia média, formada na sua maior parte de grãos de quartzo e grãos carbonáticos. O clima da área é do tipo tropical úmido com alta pluviosidade, e os meses de março a agosto compreendem os períodos de maior precipitação (SESTINI 1967; BITTENCOURT 1975). Observa-se, nas praias da orla, uma variedade de macro e microhabitats, o que favorece a colonização e o estabelecimento de diversas comunidades de organismos.

O objetivo deste trabalho foi inventariar as espécies de Echinodermata de algumas praias de Salvador e fornecer observações preliminares sobre a ecologia destas espécies.

1) Departamento de Zoologia, Instituto de Biologia, Universidade Federal da Bahia. Campus Universitário de Ondina, 40170-110 Salvador, Bahia, Brasil. E-mail: orane@ufba.br

2) Departamento de Ciências Biológicas, Universidade Estadual de Feira de Santana. Campus Universitário, BR 116, Km 03, 44031-460 Feira de Santana, Bahia, Brasil. E-mail: walter@uefs.br 


\section{MATERIAL E MÉTODOS}

\section{Área de estudo}

As características fisiográficas da área de estudo são apresentadas em BITTENCOURT (1975). As praias visitadas - Itapuã, Pituba, Amaralina, Ondina e Ribeira - estão localizadas na figura 1. A área coletada na praia de Itapuã (Fig. 2) localiza-se próxima ao Farol, e é formada por ampla área de afloramentos de arrecifes, inserida no setor de menor energia da orla, com estirâncios relativamente largos (25 a $35 \mathrm{~m}$ ao nível da baixa-mar) e declives suaves ( 2 a $\left.3^{\circ}\right)$. A praia da Pituba (Fig. 3) possui fisiografia semelhante à anterior, no entanto, está localizada próxima à desembocadura do Rio Camurujipe. A praia de Amaralina, localiza-se em um setor onde a costa muda bruscamente de direção, e as condições de energia são maiores. A praia de Ondina está situada no setor da orla de maior energia de ondas, com estirâncios de pouca largura ( 10 a $20 \mathrm{~m}$ ao nível da baixa-mar) e declives de 5 a $7^{\circ}$. Amaralina e Ondina são praias de fisiografia semelhante à Itapuã e Pituba, no entanto, com área de arrecifes menores.

Dentre as praias de Salvador banhadas pela Baía de Todos os Santos, destaca-se a Praia da Ribeira (Fig. 4), por apresentar uma variedade de organismos bentônicos sendo, inclusive, um referencial para coleta de organismos e estudos de ecologia animal por parte dos estudantes de graduação em Biologia. Possuindo substrato arenoso, e com características distintas das demais praias estudadas, não possui afloramentos de arrecifes e apresenta menor energia de ondas.

\section{Métodos}

Foram realizadas coletas qualitativas em busca de exemplares de equinodermes nas cinco praias descritas anteriormente, nos anos de 1991, 1993 e 1994, na zona entremarés.

Os exemplares foram coletados manualmente, removendo-se fragmentos de rochas e revolvendo-se o sedimento, com auxílio de uma pá, à uma profundidade de aproximadamente $10 \mathrm{~cm}$. Algas calcárias e esponjas também foram observadas em campo e, em alguns casos, amostras foram levadas ao laboratório para triagem em busca de possíveis indivíduos que a elas pudessem estar associados.

Os espécimens coletados foram acondicionados em recipientes plásticos contendo água do mar e transportados ao laboratório onde foram anestesiados com $\mathrm{MgCl}_{2}$, fixados em formol neutro a $5 \%$ durante $48 \mathrm{~h}$ e conservados em álcool glicerinado 70\% (Ophiuroidea) e álcool a 70\% (demais grupos), sendo posteriormente identificados sob microscópio estereoscópico (HENDLER et al. 1995). Utilizou-se como bibliografia básica os trabalhos de TOMMASI (1965, 1966, 1969, 1970a,b). O material encontra-se depositado na coleção do Laboratório de Echinodermata do Departamento de Zoologia, Instituto de Biologia da Universidade Federal da Bahia.

\section{RESULTADOS}

Foram obtidos 28 táxons de Echinodermata distribuídos em 19 famílias apresentadas a seguir: 


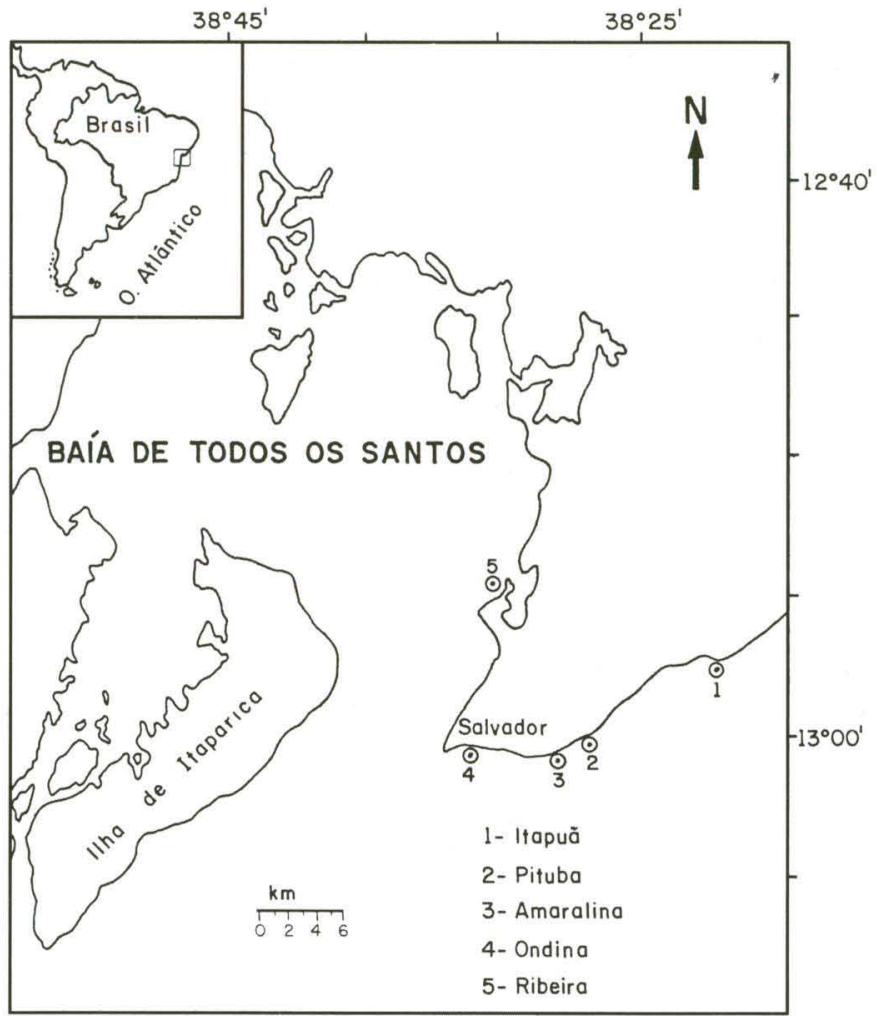

Fig. 1. Localização da área de estudo e das praias visitaaas na orla marítima de Salvador.

Asteroidea

Linckiidae

Linckia guildingi Gray, 1840

Echinasteridae

Echinaster echinophorus (Lamarck, 1816)

Ophiuroidea

Ophiomyxidae

Ophiomyxa flaccida (Say, 1825)

Amphiuridae

Amphipholis januarii Ljungman, 1867

Amphipholis squamata (Delle Chiaje, 1828)

Amphiura stimpsonii Lütken, 1859

Micropholis gracillima (Stimpson, 1852)

Ophiocnida scabriuscula (Lütken, 1859)

Ophiactidae

Ophiactis savignyi(Müller \& Troschel, 1842) 

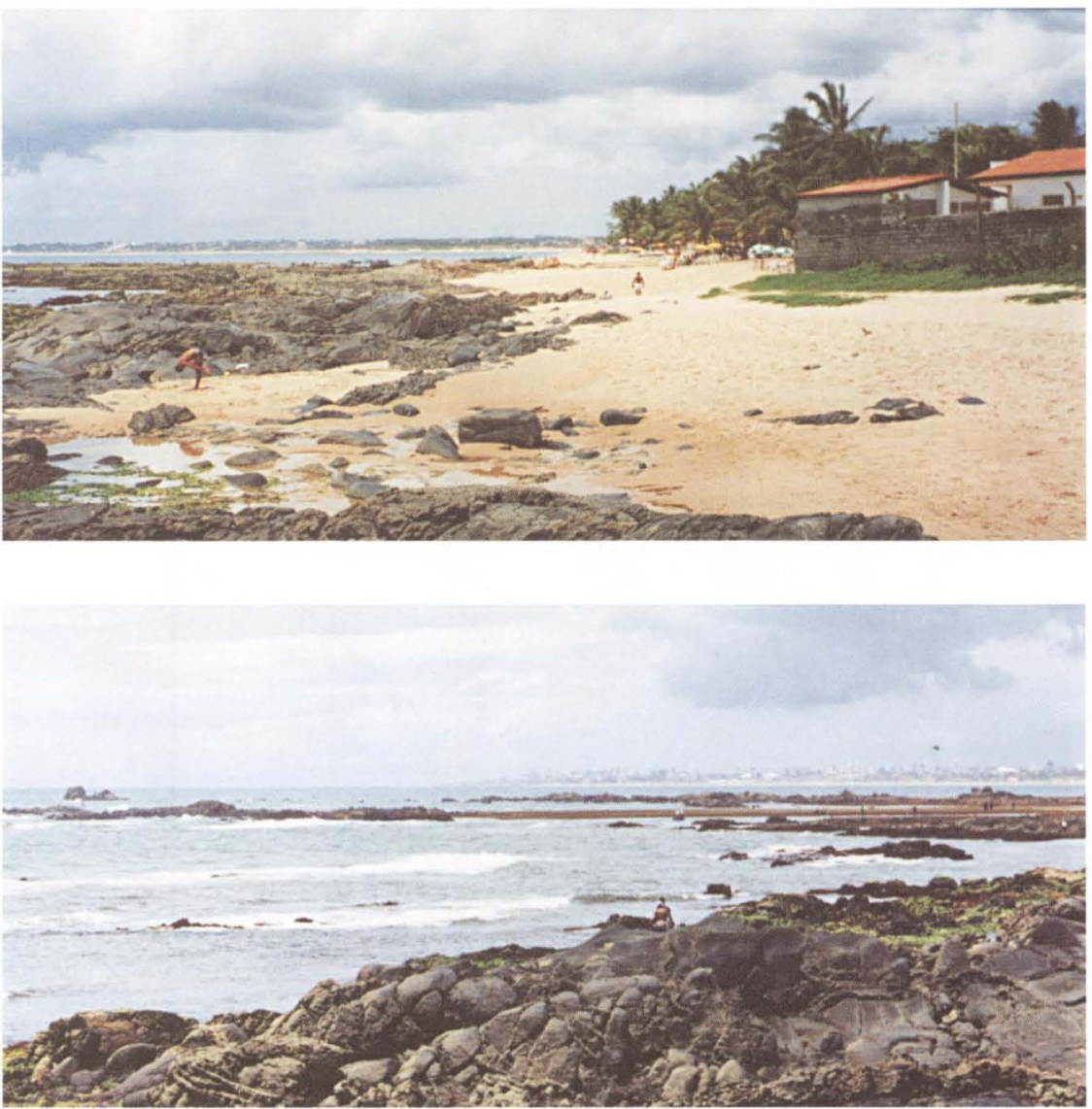

Fig. 2. Praia de Itapuã, proximidades do Farol, na baixa-mar $(0,1)$ : praia (acima) e arrecifes (abaixo). Fotos de novembro de 1999.

Ophiotrichidae

Ophiotrix angulata (Say, 1825)

Ophiodermatidae

Ophioderma apressum (Say, 1825)

Ophioderma cinereum Müller \& Troschel, 1842

Ophiocomidae

Ophiocoma echinata (Lamarck, 1816)

Ophiocoma pumila Lütken, 1859

Ophiocoma wendtii Müller \& Troschel, 1842

Ophiuridae

Ophiolepis impressa Lütken, 1859 
Ophionereididae

Ophionereis reticulata (Say, 1825)

Echinoidea

Cidaridae

Eucidaris tribuloides (Lamarck, 1816)

Diadematidae

Diadema antillarum (Philippi, 1845)

Toxopneustidae

Litechinus variegatus (Lamarck, 1816)

Tripneustes ventricosus (Lamarck, 1816)

Echinometridae

Echinometra lucunter (Lamarck, 1758)

Clypeasteridae

Clypeaster subdepressus (Gray, 1825)

Mellitidae

Encope emarginata (Leske, 1778)

Leodia sexiesperforata (Leske, 1778)

Holothuroidea

Holothuriidae

Holothuria grisea Selenka, 1867

Cucumariidae

Ocnus braziliensis (Verrill, 1868)

Crinoidea

Tropiometridae

Tropiometra carinata (Lamarck, 1816)

A ocorrência dos diversos táxons encontrados nas praias estudadas pode ser observada na tabela I.

A classe Ophiuroidea foi a que apresentou o maior número de espécies (15), seguida de Echinoidea (8), Asteroidea (2), Holothuroidea (2) e Crinoidea (1).

Analisando-se a frequência de ocorrência das classes de Echinodermata, observa-se que Ophiuroidea e Echinoidea estiveram presentes em $100 \%$ das praias visitadas, seguidas de Asteroidea e Holothuroidea (80\%) e Crinoidea (20\%).

Com relação aos táxons, pode-se observar quatro grupos no que se refere à ocorrência nas cinco praias:

Primeiro grupo. Táxons com $80 \%$ de freqüência: Ophiactis savignyi, Ophiotrix angulata, Ophioderma apressum, Ophioderma cinereum, Ophiocoma echinata, Echinometra lucunter, Eucidaris tribuloides e Ocnus braziliensis (29\% das espécies);

Segundo grupo. Táxons com $60 \%$ de freqüência: Linckia guildingi, Ophiocnida scrabriuscula, Ophiolepis impressa, Ophionereis reticulata, Tripneustes ventricosus e Holothuria grisea (21\% das espécies); 

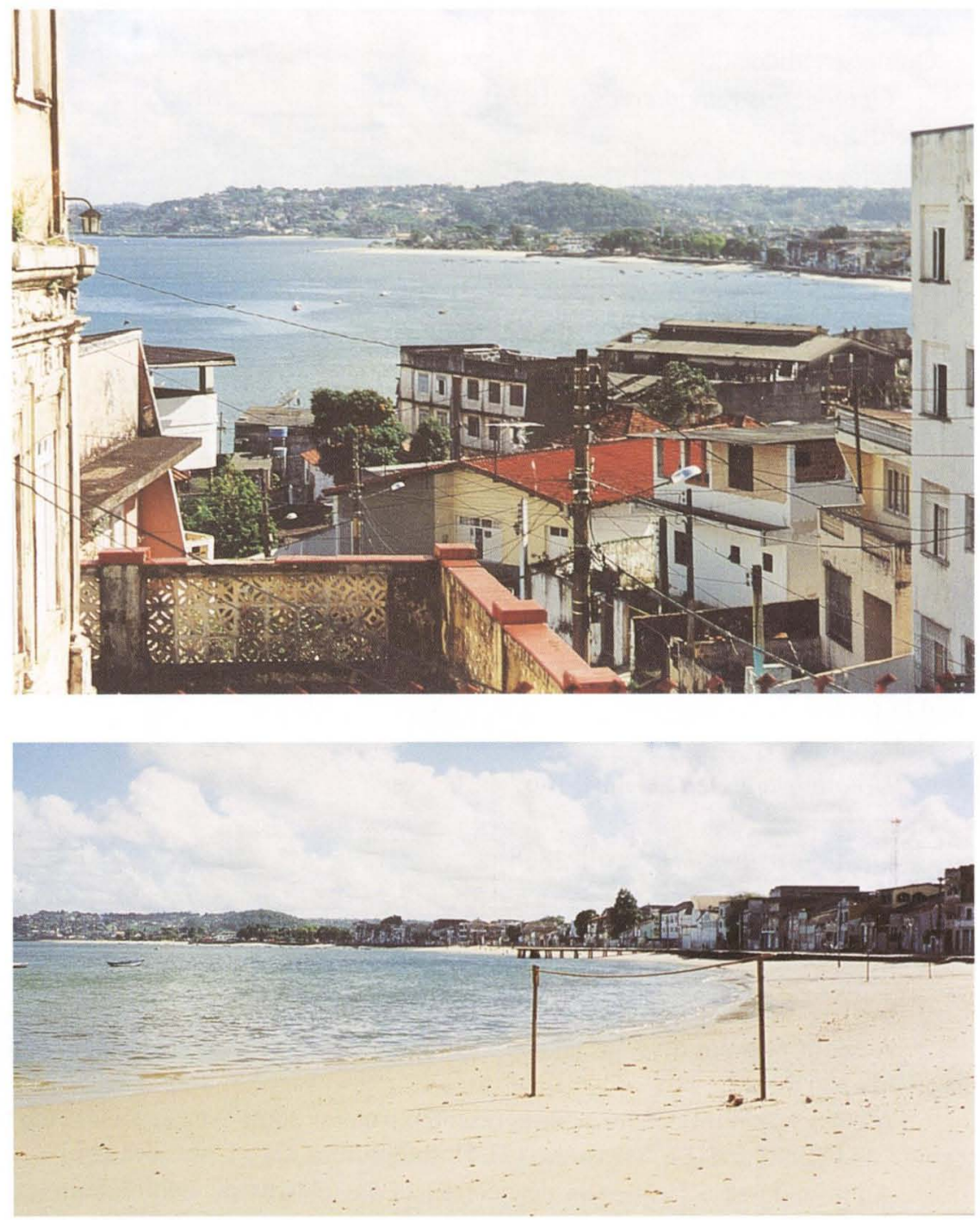

Fig. 3. Praia da Pituba, atrás do Clube Português, na baixa-mar $(0,1)$ : praia (acima) e arrecifes (abaixo). Fotos de novembro de 1999.

Terceiro grupo. Táxons com $40 \%$ de freqüência: Echinaster echinophorus, Amphipholis januarii, Amphipholis squamata, Ophiocoma pumila e Ophiocoma wendtii ( $18 \%$ das espécies);

Quarto grupo. Táxons com 20\% de freqüência: Ophiomyxa flaccida, Amphiura stimpsonii, Micropholis gracillima, Diadema antillarum, Litechinus variegatus, Leodia sexiesperforata, Encope emarginata, Clypeaster subdepressus e Tropiometra carinata ( $32 \%$ das espécies). 
Tabela I. Ocorrência dos diversos táxons de equinodermes nas cinco praias. (+) Presença, $(-)$ ausência.

\begin{tabular}{|c|c|c|c|c|c|}
\hline \multirow[t]{2}{*}{ Táxons } & \multicolumn{5}{|c|}{ Praias } \\
\hline & Itapuã & Pituba & Amaralina & Ondina & Ribeira \\
\hline \multicolumn{6}{|l|}{ Asteroidea } \\
\hline Linckia guildingi & + & + & - & + & - \\
\hline Echinaster echinophorus & + & - & - & - & + \\
\hline \multicolumn{6}{|l|}{ Ophiuroidea } \\
\hline Ophiomyxa flaccida & + & - & - & - & - \\
\hline Amphipholis januarii & + & - & - & + & - \\
\hline Amphipholis squamata & + & - & - & + & - \\
\hline Amphiura stimpsonii & + & - & - & - & - \\
\hline Micropholis gracillima & - & - & - & - & + \\
\hline Ophiocnida scrabriuscula & + & + & - & + & - \\
\hline Ophiactis savignyi & + & + & - & + & + \\
\hline Ophiotrix angulata & + & + & + & + & - \\
\hline Ophioderma apressum & + & + & + & + & - \\
\hline Ophioderma cinereum & + & + & + & + & - \\
\hline Ophiocoma echinata & + & + & + & + & - \\
\hline Ophiocoma pumila & + & - & - & + & - \\
\hline Ophiocoma wendtii & + & - & - & + & - \\
\hline Ophiolepis impressa & + & + & - & + & - \\
\hline Ophionereis reticulata & + & + & - & + & - \\
\hline \multicolumn{6}{|l|}{ Echinoidea } \\
\hline Eucidaris tribuloides & + & + & + & + & - \\
\hline Diadema antillarum & + & - & - & - & - \\
\hline Litechinus variegatus & - & - & - & - & + \\
\hline Tripneustes ventricosus & + & + & - & + & - \\
\hline Echinometra lucunter & + & + & + & + & - \\
\hline Clypeaster subdepressus & - & - & - & - & + \\
\hline Encope emarginata & - & - & - & - & + \\
\hline Leodia sexiesperforata & + & - & - & - & + \\
\hline \multicolumn{6}{|l|}{ Holothuroidea } \\
\hline Holothuria grisea & + & + & - & + & - \\
\hline Ocnus braziliensis & + & + & + & - & - \\
\hline \multicolumn{6}{|l|}{ Crinoidea } \\
\hline Tropiometra carinata & + & - & - & - & - \\
\hline Riqueza ( $n^{\circ}$ de espécies) & 24 & 14 & 7 & 17 & 7 \\
\hline
\end{tabular}

A riqueza de espécies nas praias estudadas, evidenciada na tabela I, foi maior em Itapuã (24 espécies), seguida por Ondina (17), Pituba (14) e apresentando menores valores, Amaralina e Ribeira, ambas com sete espécies.

Os diferentes habitats onde os exemplares de equinodermes foram coletados são apresentados na tabela II. Onde observa-se que a riqueza de espécies variou em função do tipo de habitat.

Amphipholis januarii, Amphipholis squamata, Amphiura stimpsonii e jovens de Ophiocoma pumila e Echinometra lucunter só foram coletadas em algas calcárias. Ophiactis savignyi e Ophiotrix angulata ocorreram tanto em algas calcárias quanto em esponjas. Linckia guildingi, Holothuria grisea, Ocnus braziliensis, Ophiomyxa flaccida, Ophiocnida scrabriuscula, Ophiotrix angulata, Ophioderma apressum, Ophioderma cinereum, Ophiocoma echinata, Ophiocoma wendtii, Ophiolepis impressa, Ophionereis reticulata e Tropiometra carinata ocorreram sob rochas e/ou em fendas de rochas e locas. Sobre a areia, ou recobertas por uma fina camada de areia, observaram-se as seguintes espécies: Echinaster echinophorus, Litechinus variegatus, Encope emarginata, Leodia sexiesperforata e Clypeaster subdepressus. 

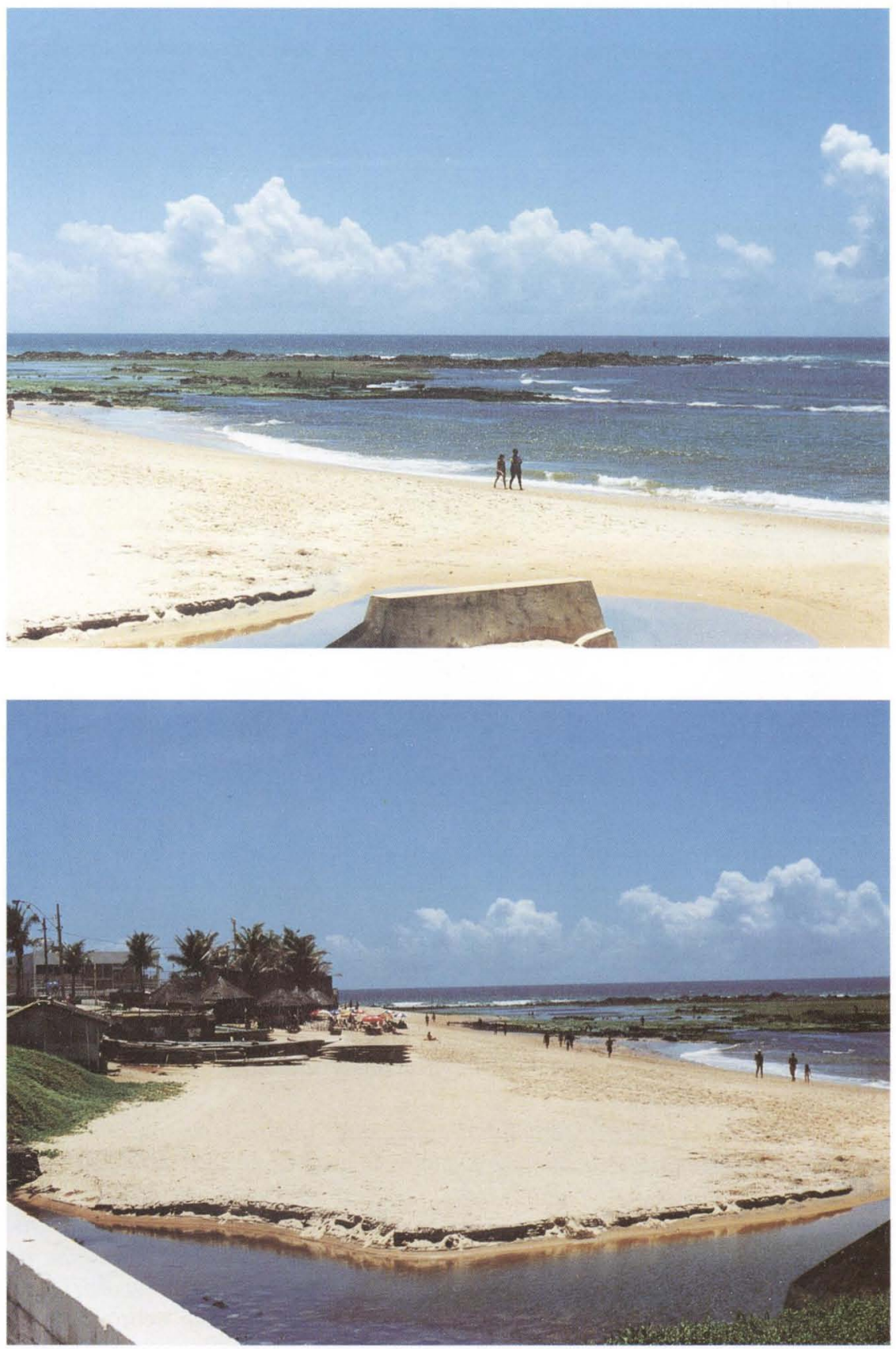

Fig. 4. Praia da Ribeira, na costa leste da Baía de Todos os Santos, início da maré vazante. Vista do alto da colina do Bonfim (acima) e trecho da praia (abaixo). Fotos de novembro de 1999. 
Tabela II. Tipos de habitats onde foram coletados exemplares de equinodermes. (1) Algas calcárias, (2) esponjas, (3) sob rochas, (4) sobre rochas, (5) em fendas de rochas, (6) em locas, (7) enterrados no sedimento, (8) sobre o sedimento.

\begin{tabular}{|c|c|c|c|c|c|c|c|c|}
\hline \multirow[t]{2}{*}{ Táxons } & \multicolumn{8}{|c|}{ Habitats } \\
\hline & 1 & 2 & 3 & 4 & 5 & 6 & 7 & 8 \\
\hline \multicolumn{9}{|l|}{ Asteroidea } \\
\hline Linckia guildingi & & & $x$ & & $x$ & $x$ & & \\
\hline Echinaster echinophorus & & & & & & $x$ & & $x$ \\
\hline \multicolumn{9}{|l|}{ Ophiuroidea } \\
\hline Ophiomyxa flaccida & & & $x$ & & & $x$ & & \\
\hline Amphipholis januarii & $x$ & & & & & & & \\
\hline Amphipholis squamata & $\begin{array}{l}x \\
x\end{array}$ & & & & & & & \\
\hline $\begin{array}{l}\text { Amphiura stimpsonii } \\
\text { Micropholis gracillima }\end{array}$ & $x$ & & & & & & $x$ & \\
\hline Ophiocnida scrabriuscula & & & $\mathrm{x}$ & & & & $\mathrm{x}$ & \\
\hline Ophiactis savignyi & $\mathrm{x}$ & $\mathrm{x}$ & & & & & & \\
\hline Ophiotrix angulata & $\mathrm{x}$ & $\mathrm{x}$ & $x$ & & $x$ & $x$ & & \\
\hline Ophioderma apressum & & & $x$ & & $x$ & $x$ & & \\
\hline Ophioderma cinereum & & & $\mathrm{x}$ & & $x$ & $x$ & & \\
\hline Ophiocoma echinata & & & $\mathrm{x}$ & & $x$ & $\mathrm{x}$ & & \\
\hline Ophiocoma pumila & $\mathrm{x}$ & & & & & & & \\
\hline Ophiocoma wendtii & $x$ & & $x$ & & & & & \\
\hline Ophiolepis impressa & & & $\mathrm{x}$ & & & & & \\
\hline Ophionereis reticulata & $x$ & & $x$ & & & & $x$ & \\
\hline \multicolumn{9}{|l|}{ Echinoidea } \\
\hline Eucidaris tribuloides & & & & $x$ & & $\mathrm{x}$ & & \\
\hline Diadema antillarum & & & & $x$ & & $\mathrm{x}$ & & \\
\hline Litechinus variegatus & & & & & & & & $x$ \\
\hline Tripneustes ventricosus & & & & $x$ & & $x$ & & $x$ \\
\hline Echinometra lucunter & $\mathrm{x}$ & & & $x$ & & $\mathrm{x}$ & & \\
\hline Clypeaster subdepressus & & & & & & & $\mathrm{x}$ & $\mathrm{x}$ \\
\hline Encope emarginata & & & & & & & $x$ & $x$ \\
\hline Leodia sexiesperforata & & & & & & & $x$ & $\mathrm{x}$ \\
\hline \multicolumn{9}{|l|}{ Holothuroidea } \\
\hline Holothuria grisea & & & $\mathrm{x}$ & & $x$ & $\mathrm{x}$ & & \\
\hline Ocnus braziliensis & & & $x$ & & $x$ & $x$ & $x$ & \\
\hline \multicolumn{9}{|l|}{ Crinoidea } \\
\hline Tropiometra carinata & & & & & & $\mathrm{x}$ & & \\
\hline Riqueza ( $n^{\circ}$ de espécies) & 9 & 2 & 12 & 4 & 7 & 14 & 7 & 6 \\
\hline
\end{tabular}

Os representantes da família Amphiuridae (Ophiuroidea) demonstraram maior capacidade de autotomização, partindo-se facilmente, tanto os braços quanto o disco, enquanto que as espécies do gênero Ophioderma e Ophiocoma echinata demonstraram muito menos autotomização. Foi observado que os ofiuróides mais resistentes (Ophiodermatidae e Ophiocoma echinata) encontravam-se geralmente sob rochas; por outro lado, os mais frágeis (Amphiuridae, Ophiocoma pumila, Ophiactis savignyi e Ophiotrix angulata) encontravam-se enterrados no sedimento, nos emaranhados de algas ou em associação com esponjas. Os Asteroidea registrados na área de estudo foram facilmente coletados, não demonstrando nenhum tipo de aderência ao substrato, da mesma forma que Clypeaster subdepressus, Encope emarginata, Leodia sexiesperforata e Litechinus variegatus, sendo que este último foi sempre encontrado recoberto por conchas vazias de moluscos. Eucidaris tribuloides e Tripneutes ventricosus também foram coletados facilmente, ao contrário de Echinometra lucunter e dos Holothuroidea, que se encontravam sempre firmemente aderidos às rochas por meio do sistema ambulacrário. 


\title{
DISCUSSÃO E CONCLUSÕES
}

A proporção do número de táxons registrados na área de estudo, bem como as freqüências de ocorrência, assemelham-se à de estudos realizados na plataforma continental, onde os Ophiuroidea foram sempre dominantes (TOMMASI 1985; TOMMASI \& ARON 1987, 1988; TOMMASI et al. 1988a,b).

De acordo com os estudos zoogeográficos realizados por PALÁCIO (1982), a fauna de equinodermos da área de estudo pode ser considerada tipicamente tropical de águas quentes. Quanto à batimetria, as espécies em questão são tipicamente litorâneas, ou possuem ampla distribuição batimétrica (TOMMASI 1965, 1966, 1969, 1970a,b).

Ophiolepis impressa, registrado para a região infralitoral por TOMMASI (1970b), ocorreu na zona entremarés das praias de Itapuã, Pituba e Ondina. No entanto, a espécie foi registrada recentemente para esta região por HENDLER et al. (1995).

A riqueza de espécies variou nas cinco praias. Itapuã e Pituba apresentam fisiografias semelhantes e estão em ambientes de menor energia da orla atlântica. No entanto, a segunda praia sofre maior ação antrópica, pela proximidade de um rio poluído e por banhistas. As praias de Ondina e Amaralina, embora próximas, apresentaram valores distintos, sendo que a segunda localiza-se em um trecho de mudança de direção da costa. E a praia da Ribeira, apresentando menor valor, sofre a influência dos subúrbios da cidade, além de possuir substratos diferentes das demais praias estudadas. Embora estes dados possam explicar a diferença nos valores de riqueza encontrados, estudos quantitativos são necessários para uma avaliação mais adequada da biodiversidade de equinodermes destes ambientes.

Os diferentes exemplares foram encontrados em tipos de habitats variados, observa-se, no entanto, que houve maior riqueza de espécies em ambientes protegidos, como locas (14 espécies) e sob rochas (12). Cabe ressaltar, também, que estudos quantitativos devem ser realizados para uma melhor avaliação da especificidade ou não das espécies e da riqueza destes habitats.

Durante as coletas para a realização deste inventário foi observada a interação entre Ophionereis reticulata e o poliqueto Malmgreniella variegata (Treadwell, 1917), como recentemente registrado para a costa brasileira por SANTA-ISABEL et al. (1996).

\begin{abstract}
AGRADECIMENTOS. Ao Biólogo Carlos José Miranda e à MSc. Cynthia Lara de Castro Manso (ambos da Universidade Federal do Rio de Janeiro - UFRJ) pela identificação e confirmação de algumas espécies. Ao Dr. Ricardo Silva Absalão e à Profa Vera Abud P. Silva (ambos da UFRJ) pelas críticas e sugestões oferecidas. Ao Biólogo Paulo Márcio Santos Costa (UFRJ) pela confecção do mapa. E ao Prof. Dr. Guilherme Lessa (Universidade Federal da Bahia) pela revisão do Abstract.
\end{abstract}

\section{REFERÊNCIAS BIBLIOGRÁFICAS}

Albuquerque, M.N. \& A. Guile. 1991. Ophiuroidea (Echinodermata) ao largo do Brasil: Banco dos Abrolhos, Cadeia submarina Vitória-Trindade e Plataforma continental adjacente. Bol. Mus. Nac., N.S. Zoologia, Rio de Janeiro, 353: 1-30.

Bittencourt, A.C. DA S.P. 1975. Sedimentação recente na Costa Atlântica de Salvador. Rev. Bras. Geoc. 5: 46-63. 
Hendler, G.; J.E. Miller; D.L. Pawson \& P.M. Kier. 1995. Sea stars, sea urchins, and alliens: echinoderms of Florida and the Caribbean. Washington, D.C., Smithsonian Institution Press, $391 \mathrm{p}$.

Lana, P. da C.; M.G. DE CAmargo; R.A. Brogim \& V.J. IsAaC. 1996. O Bentos da costa brasileira: avaliação crítica e levantamento bibliográfico (1858 - 1996). Rio de Janeiro, REVIZEE, MMA/CIRM/FEMAR, 432p.

Manso, C.L.C. 1993. Ofiuróides da Plataforma Brasileira. Parte II: Norte do Estado do Rio de Janeiro, Estado do Espírito Santo, Sul do Estado da Bahia e Bancos Royal Charlott, Hotspur e Davis (Echinodermata: Ophiuroidea). Rev. Brasil. Biol. 53 (2): 189-195.

PALÁC1O, F.J. 1982. Revisión Zoogeográfica Marina del Sur del Brasil. Bolm. Inst. Oceangr. Univ. São Paulo, 31 (1): 69-92.

Rathbun, R. 1879. A list of the brazilian echinoderms with notes on their distribution. Trans. Conn. Acad. Arts Sci., New Haven, 5: 139-158.

SantA-IsAbel, L.M. DE; W.R.P. Cerqueira \& O.F.S. DE S. Alves. 1996. Associação entre Ophionereis reticulata (Say) (Ophiuroidea, Ophionereididae) e Malmgreniella variegata (Treadwell) (Polychaeta, Polynoidae) das Praias de Salvador, Bahia, Brasil. Revta bras. Zool. 13 (1): 137-142.

SEstinı, G. 1967. Textural characteres of Salvador beach sands. Bol. Univ. Fed. Paraná, Curitiba, 8: $1-15$.

Tomması, L.R. 1965. Lista dos Crinóides recentes do Brasil. Contr. Inst. Oceanogr. Univ. São Paulo, Sér. Oceanogr. Biol. (9): 1-33.

. 1966. Lista dos Equinóides recentes do Brasil. Contr. Inst. Oceanogr. Univ. São Paulo, Sér. Oceanogr. Biol., (11): 1-50.

. 1969. Lista dos Holothuroidea recentes do Brasil. Contr. Inst. Oceanogr. Univ. São Paulo, Sér. Oceanogr. Biol. (15): 1-50.

-1970a. Lista dos Asteróides recentes do Brasil. Contr. Inst. Oceanogr. Univ. São Paulo, Sér. Oceanogr. Biol. (18): 1-61.

-1970b. Os Ofiuróides recentes do Brasil e regiões vizinhas. Contr. Inst. Oceanogr. Univ. São Paulo, Sér. Oceanogr. Biol. (20): 1-146.

- 1985. Equinodermes da Região da Ilha da Vitória (SP). Relat. Int. Inst. Oceanogr. Univ. São Paulo (13): 1-4.

TOMmASI, L.R. \& M.A. ARON. 1987. Equinodermes dos bancos submarinos da cadeia de montanhas Vitória-Trindade. Relat. Int. Inst. Oceanogr. Univ. São Paulo (18): 1-9.

- 1988. Equinodermes da plataforma continental do Sudeste do Estado da Bahia. Relat. Int. Inst. Oceanogr. Univ. São Paulo (19): 1-6

TOMMASI, L.R.; S.M. CASTRO \& E.C.P.M. SoUS^. 1988a. Echinodermata coletados durante as campanhas oceanográficas do N/Oc. "Almirante Saldanha" no Atlântico Sul Ocidental. Relat. Int. Inst. Oceanogr. Univ. São Paulo (21): 1-11.

TOMmASI, L.R.; M.C.W. CERnEA \& M.C.G. CondeiXA. 1988b. Equinodermes coletados pelo N./Oc. “Almirante Saldanha”, entre 265' S e 38³9'S. Relat. Int. Inst. Oceanogr. Univ. São Paulo (22): $1-11$.

VerRILL, A.E. 1868. Notes of the radiate on the Museum of Yale College, with descriptions of genera and species. 4. Notice on the corals and echinoderms collected by Prof. C.F. Hartt at the Abrolhos Reefs, Province of Bahia, Brazil, 1867. Trans. Conn. Acad. Arts Sci. 1 (2): 351-371.

Recebido em 22.X.1998; aceito em 18.V.2000. 